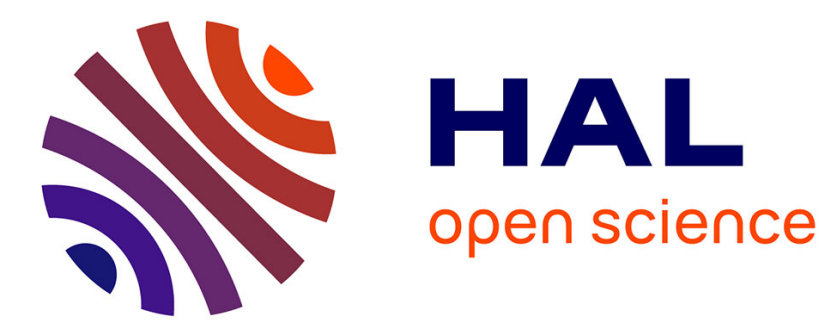

\title{
Traitement de surface en continu avec un laser Nd:YAG impulsionnel
}

\author{
F. Lemoine, D. Grevey, I. Vastra, A. Vannes
}

\section{To cite this version:}

F. Lemoine, D. Grevey, I. Vastra, A. Vannes. Traitement de surface en continu avec un laser Nd:YAG impulsionnel. Journal de Physique IV Proceedings, 1994, 04 (C4), pp.C4-43-C4-46. 10.1051/jp4:1994407 . jpa-00252545

\section{HAL Id: jpa-00252545 https://hal.science/jpa-00252545}

Submitted on 1 Jan 1994

HAL is a multi-disciplinary open access archive for the deposit and dissemination of scientific research documents, whether they are published or not. The documents may come from teaching and research institutions in France or abroad, or from public or private research centers.
L'archive ouverte pluridisciplinaire HAL, est destinée au dépôt et à la diffusion de documents scientifiques de niveau recherche, publiés ou non, émanant des établissements d'enseignement et de recherche français ou étrangers, des laboratoires publics ou privés. 


\title{
Traitement de surface en continu avec un laser Nd:YAG impulsionnel
}

\author{
F. LEMOINE $^{*, * *}$, D.F. GREVEY ${ }^{* *}$, I. VASTRA ${ }^{* * *}$ et A.B. VANNES* \\ * CALFETMAT, MMP, Ecole Centrale de Lyon, BP. 131, 69131 Ecully, France \\ ** LTm, IUT, 12 rue de la Fonderie, 71200 Le Creusot, France \\ ${ }^{* * *}$ Centre Technique, FRAMATOME, 3 rue du Guide, 71200 Le Creusot, France
}

\begin{abstract}
This work presents the results of investigations in case of laser cladding with a pulsed Nd:YAG laser. We discuss about the experimental parameters in view to realize single clad and particulary the laser data (energy, frequency, pulse duration). The main results are the clad formation beyond an energy threshold and beyond a threshold pulse duration, the clad behaviour is the same as a clad obtained with a cw laser. Thanks to a cross-section modeling, we compare calculations with experiments and we conclude that the interaction efficiency depends widely on powder spray characteristics.
\end{abstract}

\section{I - Introduction}

Dans cette étude, nous présentons et discutons de résultats de dépôts de stellite obtenus par projection de poudre dans un faisceau Nd:YAG impulsionnel. Nous passons en revue l'influence sur les cordons des différents paramètres liés à la nature impulsionnelle de l'émission: l'énergie des impulsions $(E)$, leur durée $(\tau)$ et leur fréquence $(F)$. En dernier lieu, nous présentons pour mieux comprendre les phénomènes, un modèle permettant de prévoir la section de ces cordons.

Le laser utilisé est un laser impulsionnel pouvant délivrer une puissance moyenne de $1000 \mathrm{~W}$ et $10 \mathrm{~kW}$ de puissance crête. Le faisceau est transporté par une fibre optique à saut d'indice de $1000 \mu \mathrm{m}$ de diametre et d'ouverture numérique 0,22. L'injection de la poudre dans le faisceau se fait de façon continue et latérale au faisceau. La poudre est de la stellite (composition moyenne $=60 \% \mathrm{Co}, 25 \% \mathrm{Cr}, 5 \%$ $\mathrm{W}, 1,5 \% \mathrm{C})$ de granulométrie comprise entre 50 et $150 \mu \mathrm{m}$ que nous avons tamisé par plage de $20 \mu \mathrm{m}$. Le substrat est un acier inoxydable (Z3CN17-10) sur lequel nous réalisons de simples cordons dits monopasse dont nous discutons de l'évolution de la section (figure 1).

\section{II - Résultats et discussion}

- Energie des impulsions:

Toutes choses étant égales par ailleurs, lorsque l'énergie des impulsions varie la puissance crête $\left(P_{c}=E / \tau\right)$ et la puissance moyenne $\left(P_{m}=E . F\right)$ varient elles aussi. Nous pouvons comparer la section de dépôts obtenus dans les deux cas suivants:

i) L'énergie $E$ varie avec les puissances crête et moyenne constantes (figure 2).

ii) L'énergie $\mathrm{E}$ varie ainsi que les puissances crête et moyenne (figure 3 ).

Le taux de recouvrement est identique et constant dans les deux cas (99\%).

Dans les deux cas et ceci malgré une variation des parametres dépendants du jet de poudre, les résultats sont similaires et peuvent se superposer (rendement d'interaction identique). On peut dire qu'il n'y a pas de modification de la section des dépôts si on travaille à puissances constante (fréquence et durée des impulsions variables) ou variable car seule l'énergie de l'impulsion est prépondérante sur le 
résultat. Pour que dans ces conditions le dépôt se forme, Il est nécessaire de dépasser une énergie minimale.

- Influence de la fréquence (taux de recouvrement):

La fréquence va intervenir sur le taux de recouvrement des impulsions et donc jouer sur la quantité de poudre déposée par unité de longueur. D'autre part suivant la fréquence, on peut penser que le bain fondu se déplace de façon continue ou discontinue. Dans ce cas précis, la durée d'impulsion joue un rôle déterminent.

\section{- Durée des impulsions:}

En fonction de la durée des impulsions (figure 4), la section des dépôts augmente jusqu'à atteindre une section constante à partir d'une durée d'impulsion supérieure à $3 \mathrm{~ms}$. Au delà de cette durée, que nous appelons durée d'impulsion seuil ( $\tau$ seuil), on montre que le dépôt se comporte comme un dépôt "continu" car il y a égalité des rendements de poudre (masse de dépôt/masse de poudre projetée) entre celui déterminé pour une source continue et celui déterminé pour la source impulsionnelle.

Nous pouvons chercher à déterminer la durée d'impulsion seuil en posant comme hypothèses que pendant le temps mort, $t_{m}$, (durée entre deux impulsions) une partie de la poudre est fondue par le bain liquide et que celui-ci poséde une certaine énergie. C'est à dire que pendant la durée de l'impulsion le bain liquide est porté à une température importante pouvant aller de la température ambiante $\left(T_{a}\right)$ à la température de vaporisation $\left(T_{v}\right)$. Le bilan énergétique est le suivant:

$$
E_{1}=E_{2}+E_{3}
$$

avec: $E_{1}=$ énergie du bain acquise par l'échauffement de la poudre pendant la durée d'impulsion; $E_{2}=$ énergie nécessaire à la fusion de la poudre projetée durant la durée d'impulsion; $E_{3}=$ énergie nécessaire à la fusion de la poudre projetée pendant le temps mort. Le traitement sera continu si le surplus d'énergie acquis par le bain en fusion $\left(E_{1}-E_{2}\right)$ est supérieur à l'énergie $\left(E_{3}\right)$. L'expression de la durée d'impulsion seuil est ainsi:

$\tau_{\text {seuil }}=t_{m} \cdot \frac{\left[C_{p}\left(T_{f}-T_{a}\right)+L_{f}\right]}{\left[C_{p}\left(T_{v}-T_{a}\right)+L_{f}+L_{v}\right]-\left[C_{p}\left(T_{f}-T_{a}\right)+L_{f}\right]} \quad$ avec $\quad \tau_{\text {seuil }}+t_{m}=1 / F$

Cette durée d'impulsion à partir de laquelle nous obtenons un régime continu dépend uniquement des caractéristiques physiques de la poudre $\left(C_{p}\right.$ : chaleur spécifique; $\mathrm{T}_{\mathrm{f}}$ : température de fusion; $\mathrm{L}_{\mathrm{f}}$ : chaleur latente de fusion; $\mathrm{L}_{\mathrm{v}}$ : chaleur latente de vaporisation) et de la fréquence des impulsions. Dans notre cas de figure, avec une fréquence de $50 \mathrm{~Hz}$, cette durée est de $2 \mathrm{~ms}$.

\section{V- Modélisation de la section des cordons}

Il est possible de donner une expression de la section des cordons à partir de la loi de Joule et en assimilant le jet de poudre à un nuage de particules dans lequel l'atténuation de l'onde suit une loi de Beer-Lambert [1]. A la longueur d'onde du rayonnement $\mathrm{Nd}: \mathrm{YAG}$, les phénomènes de diffusion de la lumière par les particules sont décrits par les lois de l'optique géométrique et sont ainsi dépendants de la taille des particules [2]. Par extension du modèle relatif à l'émission continue [3] et en tenant compte de la géométrie de la buse et de la discontinuité de l'émission, la section (S) des cordons peut-être donnée par:

$$
\mathrm{S}=\text { So }[1-\exp (-\mathrm{B} . \mathrm{C})]
$$


avec: $i)$ So $=$ section théorique $=\frac{E \cdot F}{V_{t} \cdot \rho\left(C_{p} \Delta T+L_{f}\right)}$

avec $\Delta T=$ variation de température $\left(T_{f}-T_{a}\right), \rho=$ densité de la poudre, $V t$ : vitesse de travail.

ii) $[1-\exp (-B$. C) $]=$ rendement d'interaction

$$
B=\text { terme lié à la poudre et à la buse }=\frac{3 R}{2 \pi \cdot V_{p} \cdot \rho \cdot r_{p} \cdot \cos \alpha\left(r_{b}+\operatorname{tg} \theta \frac{z_{1}}{\cos \alpha}\right)}
$$

Vp: vitesse des particules, $\mathrm{r}_{\mathrm{p}}$ : rayon des particules, $\alpha$ : angle entre l'axe du faisceau et l'axe $d u$ jet de poudre, $\theta$ : demi-angle de divergence du jet de poudre, $r_{b}$ : le rayon de la buse, $z_{1}$ : distance comprise entre la buse et la partie supérieure du jet de poudre.

$$
C=\text { terme lié à la géométrie du jet de poudre }=\frac{D_{p}\left(d_{b p}-z_{1}\right)}{\left(r_{b}+\operatorname{tg} \theta \frac{D_{b p}}{\cos \alpha}\right)}
$$

Dp: débit de poudre, $d_{b p}$ : distance entre la buse et le substrat.

La figure 5 montre la variation de la section en fonction du débit de poudre et compare les valeurs expérimentales et calculées. Dans le cas de la variation de la section en fonction de la durée d'impulsion, l'équation (3) donne une section calculée voisine de celle obtenue au delà de la durée d'impulsion seuil. En deçà, il faut corriger cette section par le rapport $\tau / \tau_{\text {seuil }}$ (figure 4 ).

\section{IV - Conclusions}

Cette étude a permis de mettre en évidence que des opérations de revêtements sont envisageables avec des lasers à émission impulsionnelle et que sous certaines conditions au delà d'une durée d'impulsion seuil, qui est fonction de la fréquence et des caractéristiques de la poudre, le cordon se comporte comme un cordon continu et que nous sommes dans les conditions optimales d'utilisation.

D'autre part pour que le dépôt s'initie à la surface du matériau à revêtir, il est nécessaire d'utiliser une énergie minimale par impulsion. C'est ainsi que nous définissons une énergie seuil qui est de $7 \mathrm{~J}$ dans le cas d'un dépôt de stellite sur un acier inoxydable.

La prévision de la section des cordons fait ressortir, par l'intermédiaire du rendement d'interaction, l'importance du contrôle et de la connaissance du jet de poudre, et qu'une augmentation du rendement du système passe par l'optimisation de celui-ci.

\section{Références}

1 - Lagain P. Contribution expérimentale aux traitements de surface par laser avec apport de poudre. Thèse: Doctorat: Université Aix-Marseille, (1989).

2 - Kastler, A. La diffusion de la lumière par les milieux troubles. Paris: Hermman, (1952), $77 \mathrm{p}$.

3 - Lemoine, F. \& al. Modélisation de la section de dépôts obtenus par fusion d'une poudre métallique projetée dans un faisceau laser Nd:YAG. J. Phys. III, 3 (octobre1993), p. 2043-2052. 


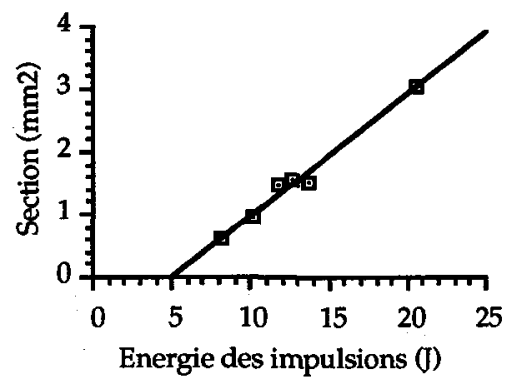

Figure 2: Variation de la section en fonction de l'énergie des impulsions. $\left(P_{m}=830 \mathrm{~W}\right.$, $P_{C}=3,8 \mathrm{~kW}$, Distance buse-piece $=15 \mathrm{~mm}$, Poudre $=50-150 \mu \mathrm{m}$, Débit de poudre $=40 \mathrm{~g} / \mathrm{mn}$ )

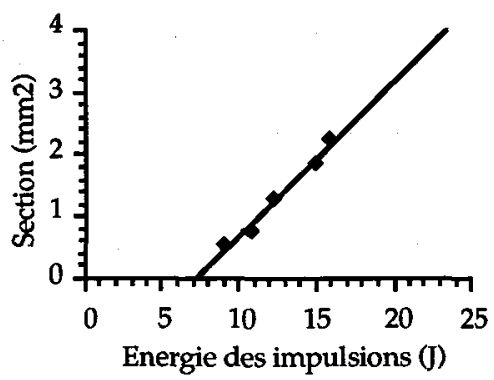

Figure 3: Variation de la section en fonction de l'énergie des impulsions. $(F=50 \mathrm{~Hz}, \tau=5 \mathrm{~ms}$, Distance buse-pièce $=17 \mathrm{~mm}$, Poudre $=50-63 \mu \mathrm{m}$, Débit de poudre $=25 \mathrm{~g} / \mathrm{mn}$, Vitesse $=150 \mathrm{~mm} / \mathrm{mn}$ )

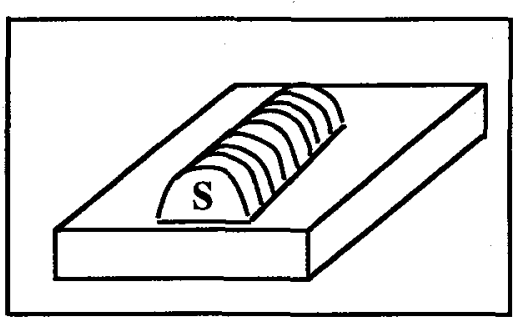

Figure 1: Schéma d'un cordon avec la section (S) analysée.

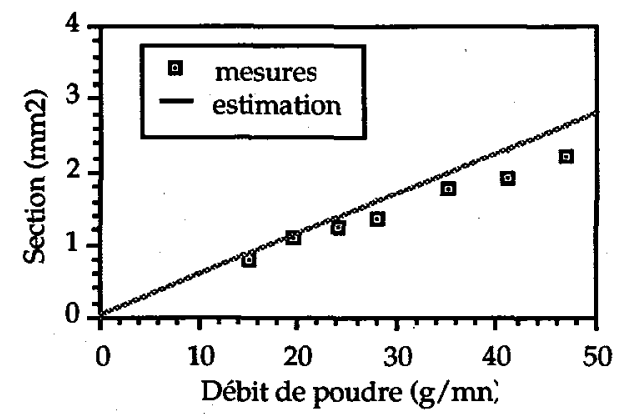

Figure 5: Variation de la section des dépôts en fonction du débit de poudre. Comparaison entre le modèle et les valeurs mesurées. ( $E=15,5 \mathrm{~J}$, $=50 \mathrm{~Hz}, \tau=4 \mathrm{~ms}, \mathrm{dbp}_{\mathrm{bp}}=17 \mathrm{~mm}, \mathrm{Vt}=210 \mathrm{~mm} / \mathrm{mn}$, Poudre $50 / 63 \mu \mathrm{m}$ )

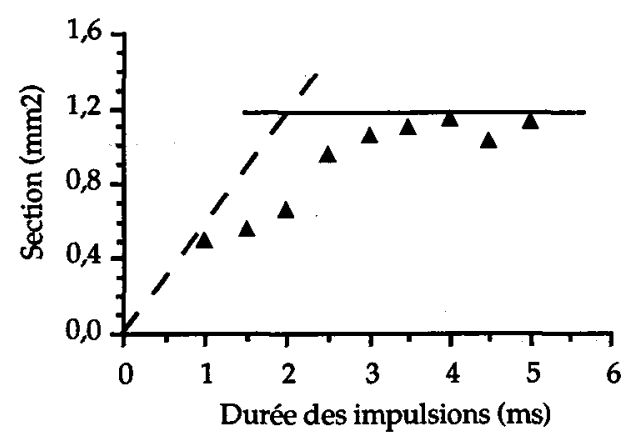

(a)

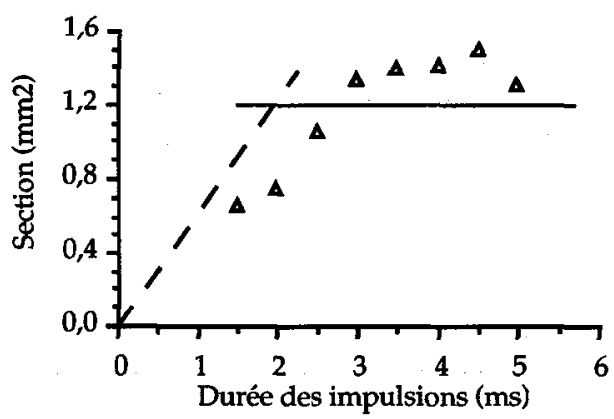

(b)

Figure 4: Variation de la section en fonction de la durée d'impulsion pour deux granulométries (a) 63-80 $\mu \mathrm{m}$ et (b) 90-112 $\mu \mathrm{m}$. Comparaison entre les mesures et le modèle au delà (-) et en deçà (- -) de la durêe d'impulsion seuil calculée( $2 \mathrm{~ms})$. 